\title{
Immune Thrombocytopenia Purpura (ITP) with Grave's Disease, in a Plasmodium Vivax Positive Patient. A Case Report
}

\author{
Syed Mohammad Mazhar Uddin', Aatera Haq1, Zara Haq², \\ Uzair Yaqoob $^{3 *}$ \\ ${ }^{1}$ Civil Hospital Karachi, Karachi, Pakistan \\ ${ }^{2}$ Dow University of Health Sciences, Karachi, Pakistan \\ ${ }^{3}$ Sindh Medical College, Dow University of Health Sciences, Karachi, Pakistan \\ Email: *ozair_91393@hotmail.com
}

How to cite this paper: Uddin, S.M.M., Haq, A., Haq, Z. and Yaqoob, U. (2018) Immune Thrombocytopenia Purpura (ITP) with Grave's Disease, in a Plasmodium Vivax Positive Patient. A Case Report. Case Reports in Clinical Medicine, 7, 146-153. https://doi.org/10.4236/crcm.2018.72012

Received: January 24, 2018

Accepted: February 10, 2018

Published: February 13, 2018

Copyright $\odot 2018$ by authors and Scientific Research Publishing Inc. This work is licensed under the Creative Commons Attribution International License (CC BY 4.0).

http://creativecommons.org/licenses/by/4.0/

\begin{abstract}
Hyperthyroidism and thrombocytopenia have long been associated with each other. We present a case of 38 years old female presenting with complaints of bleeding from gums, bleeding per rectum, hematuria and easy bruising since 7 days ago. She also had a diffuse, non-tender neck swelling moving with deglutition and positive bruit on auscultation. Her laboratory results indicated thrombocytopenia $\left(22 \times 10^{\wedge} 9 / \mathrm{L}\right)$ and her thyroid function test revealed TSH of $0.01 \mathrm{mIU} / \mathrm{ml}$ (normal: 0.17 - 4.05), free $\mathrm{T} 3$ of $19.19 \mathrm{pg} / \mathrm{ml}$ (normal: 1.6 3.7), free $\mathrm{T} 4$ of $4.09 \mathrm{ng} / \mathrm{dl}$ (normal: 0.89 - 1.79). Thyroid scan showed diffuse goiter with increased tracer uptake. Furthermore, her serum anti-thyroglobulin and anti-thyroid peroxide were elevated to $205 \mathrm{IU} / \mathrm{ml}$ and $713 \mathrm{IU} / \mathrm{ml}$ respectively. She was started on carbimazole and methylprednisolone immediately. On the 3rd day of admission, she was tested to be positive for Plasmodium Vivax after a fever spike and was started on Chloroquine (CQ) and later shifted to Primaquine (PQ). During 2 weeks of admission, her platelet count kept fluctuating between $<10 \times 10^{\wedge} 9 / \mathrm{L}$ and $<100 \times 10^{\wedge} 9 / \mathrm{L}$ with frequent transfusions of mega units of platelets. During 3rd week, her platelets improved and she was discharged with a platelet count of $370 \times 10^{\wedge} 9 / \mathrm{L}$. This case, therefore, supports the association between autoimmune thyroid diseases such as Graves' disease and idiopathic thrombocytopenic purpura (ITP) strongly suggesting the need for evaluating thyroid disease in cases of severe thrombocytopenia especially those refractory to treatment. Also, the effectiveness of treatment of thyroid disease on thrombocytopenia is also highlighted. In addition, it showed the possible added exacerbating effects of malarial infection on thrombocytopenia.
\end{abstract}




\section{Keywords}

Immune Thrombocytopenia Purpura, Malaria, Hyperthyroidism

\section{Introduction}

Hyperthyroidism and thrombocytopenia have long been associated with one another [1]. Since then, numerous studies have evaluated this relationship and the possible etiology behind [2]. Autoimmune thyroid disorders like Graves' disease and Hashimoto's thyroiditis have been documented in patients with Idiopathic Thrombocytopenic Purpura (ITP). ITP with an associated autoimmune disease has been described as secondary ITP. There are several theories that explain the low platelet count in these thyroid disorders. Autoimmunity between thyroid and platelets is considered as a predictable pathogenesis [1] [2] [3] [4]. Studies indicate that there may also be an excessive destruction of platelets as a result of increased thyroid hormones leading to activation of the reticuloendothelial system [1] [2] [4]. Genetic predisposition has also been regarded as one of the possibilities [1].

Our case also reports the likely association between Graves's disease and ITP and the exacerbating effects of hyperthyroidism on platelet count in a patient with Plasmodium Vivax positive.

\section{Case}

We describe a 38-year-old female patient, presented to the emergency department with diarrhea and vomiting since 10 days, bleeding from gums, bleeding per rectal, hematuria and easy bruising since 7 days. The patient also reported a prominent swelling in her neck which she noticed 2 days prior to her ER visit. The patient documented some symptoms of hyperthyroidism including an increase in appetite, undocumented weight loss, increased sweating, palpitations and oligomenorrhea for weeks. The past medical history was non-significant, however, in her past surgical history; she had cholecystectomy 10 years back. On examination, her vital signs showed the Blood pressure of 100/70 (normal: 120/80), a pulse of 135 beats/minute (normal: 60 - 100 beats per minute), a temperature of $98.2 \mathrm{~F}$ (normal: $98.6 \mathrm{~F}$ ), and respiratory rate of 22 breaths/minute (12 - 20 breaths per minute). On general examination, the patient had bruises/ecchymosis all over her body. She had a swelling on her neck, which was diffuse, non-tender, approximately $4 \times 4 \mathrm{~cm}$, moving with deglutition, and there was a positive thyroid bruit on auscultation. Based on this her eye examination was done and only Stellwag sign was positive. Her abdominal exam revealed splenomegaly up to $2 \mathrm{~cm}$. Rest of the examination was normal.

Upon her arrival on 6th August 2017, her complete blood count (CBC) showed hemoglobin $(\mathrm{Hb})$ of $8.9 \mathrm{~g} / \mathrm{dl}$ (normal: 11.1 - 14.5), Mean Corpuscular Volume (MCV) of $77.5 \mathrm{fL}$ (normal: 76 - 96), WBC count of 7.2* 10E9/L (normal: 
4 - 10) and platelets were $22 \times 10^{\wedge} 9 / \mathrm{L}$ (normal: $150-400$ ). Her peripheral film indicated anisocytosis, hypochromia, mild polychromasia and low platelets. Her reticulocyte count came out to be $3.5 \%$. These findings indicated anemia with a possible bone marrow response and thrombocytopenia. Her thyroid function test revealed TSH of $0.01 \mathrm{mIU} / \mathrm{ml}$ (normal: 0.17 - 4.05), free T3 of $19.19 \mathrm{pg} / \mathrm{ml}$ (normal: 1.6 - 3.7), free T4 of $4.09 \mathrm{ng} / \mathrm{dl}$ (normal: 0.89 - 1.79), her urine detailed report showed $\mathrm{Hb}+5$ and $\mathrm{RBC}>20 / \mathrm{Hpf}$. Thyroid scan was performed after intravenous injection of Tc99 m-Pertechnetate and it revealed diffuse goiter with increased tracer uptake. Furthermore, her serum anti-thyroglobulin and anti-thyroid peroxide were elevated to $205 \mathrm{IU} / \mathrm{ml}$ and $713 \mathrm{IU} / \mathrm{ml}$ respectively. Hence, with the findings consistent with hyperthyroidism, we started the patient on carbimazole $30 \mathrm{mg}$ daily. Suspecting ITP alongside hyperthyroidism, we also started IV methylprednisolone 1 gram for 5 days. On the 2 nd day of admission, her $\mathrm{CBC}$ showed platelets improving to $83 \times 10^{\wedge} 9 / 1$. However, on the 3 rd day of admission a fever spike of $100 \mathrm{~F}$ was documented and since our region is prone to malaria, the patient was tested and it came out positive for Plasmodium Vivax. Immediately the patient was started on chloroquine (CQ) $250 \mathrm{mg}$ (4 tablets stat, then 2 tabs after 6 hours and then 1 tab twice a day for two days). On her 3 rd day of admission, her platelets count fell to $4 \times 10^{\wedge} 9 / 1$, therefore the patient was transfused with a mega unit of platelets. At this point, we assumed the low platelets could be due to ITP secondary to hyperthyroidism or/and malaria secondary to Plasmodium Vivax. On the 4 th day of admission, her platelets rose to $77 \times 10^{\wedge} 9 / 1$ temporarily, before falling to $7 \times 10^{\wedge} 9 / 1$ the next day. Her 5 doses of IV methylprednisolone were completed, now she was started on prednisolone 60 mg orally, with a gradual tapering off dose. After completion of her chloroquine doses, she was tested for g6pd levels which came out to be $7.3 \mathrm{U} / \mathrm{GHb}$ (6.7 14.3 ) and hence was started on primaquine (PQ) $30 \mathrm{mg}$ daily for 14 days. She was transfused with megaunits during this time period multiple times but her platelet count remained low. On 14th August 2017, her platelets were $27 \times 10^{\wedge} 9$.

To rule out secondary causes of ITP, we tested the patient for HIV, Hepatitis B, Hepatitis C, H pylori and CMV, all came out to be negative. Her Ultrasound of the abdomen showed only mild splenomegaly. At this point in time, her malarial parasite became negative. Her ANA, ASMA, AMA, ANTI DS DNA antibodies, ENA profile and Coombs test were negative as well. Bone marrow was also normal with adequate megakaryocytes. On 17th August 2017, her platelets fell down to $5 \times 10^{\wedge} 9 / 1$, which led her to be transfused again. During the first 2 weeks of her admission, her platelet count wasn't improving and fluctuating. However, on $20^{\text {th }}$ August 2017, her platelet count improved to $300 \times 10^{\wedge} 9 / 1$, with symptomatic improvements. Since her condition improved, the patient was discharged on 22nd August 2017 with CBC showing Hb of 9 g/dl, Mcv of 86.8 and platelets of $370 \times 10^{\wedge} 9$ along with medications including carbimazole $30 \mathrm{mg}$ daily, primaquine $30 \mathrm{mg}$ (remaining doses), remaining tapering doses of prednisolone and omeprazole $20 \mathrm{mg}$ twice daily. Furthermore, the patient was asked to follow weekly on OPD basis. 


\section{Discussion}

In $50 \%$ to $80 \%$ hyperthyroidism, Graves' disease represents the intrinsic cause which is mainly attributed to the activation of TSH receptors by the circulating IgG antibodies. The result is hypertrophy and hyperplasia of thyroid follicles, thereby resulting in thyroid enlargement. The thyroid functioning test reveals a suppressed serum TSH and a raised serum T3 and T4 levels. Serum T3 is usually more elevated as compared to serum T4. Furthermore, thyroid bruit on auscultation is also a part of primary diagnostic consideration [5]. Our patient also reported an enlarged thyroid gland, followed by thyroid bruit on auscultation, with a serum $\mathrm{t} 3$ level more raised than serum $\mathrm{t} 4$. Antibody titers can also be useful. More than $95 \%$ of patients have Thyroid peroxidase antibodies (TPO) and about $50 \%$ have positive anti-thyroglobulin (TG) antibodies. In addition, antibodies to TSH receptor strongly support Graves' disease [6]. Since antibodies to TSH receptor were not available in our setup, we had to rely on the presence of Thyroid peroxidase antibodies and thyroglobulin antibodies for the diagnosis of Graves' disease. A Thyroid scan, using either radioactive iodine or Tc99m-Pertechnetate, can also be fruitful for diagnosing Graves' disease, showing enlarged gland with diffuse activity throughout the gland [7]. The use of Tc99m-Pertechnetate in thyroid scanning holds advantages of better image quality, faster procedure and lesser radiation dose as compared to radioiodine [8]. Therefore, an increased tracer uptake along with diffuse goiter in this case further aided the diagnosis. Grave's ophthalmopathy manifested as proptosis, periorbital edema, exposure keratitis, extraocular muscle infiltration, lid lag, lid retraction, Stellwag's sign (incomplete and infrequent blinking), Mobius sign (poor convergence), optic neuropathy is clinically visible in about $30 \%$ to $50 \%$ patients with the disease, but is detected in more than $80 \%$ of patients who undergo orbital imaging [5] [9]. Apart from the stellwag sign, no other signs were clinically apparent in this patient, and no orbital scan was done due to lack of signs of ophthalmopathy.

Immune thrombocytopenia (ITP) is an acquired, persistent, autoimmune-mediated destruction of platelets with counts falling less than $100 \times$ $10^{\wedge} 9 / \mathrm{L}$. It is caused by antibodies against platelets surface antigen and is presumed when there is isolated thrombocytopenia without any abnormality in white blood cells, red blood cells or peripheral blood smear. Furthermore, for the diagnosis of ITP, it is important to rule out the other possible causes of thrombocytopenia including infections by Hepatitis B, Hepatitis C virus, HIV, drugs, and diseases like Systemic lupus erythematosus and Antiphospholipid syndrome [10] [11]. Symptomatically, ITP can vary from being asymptomatic to life-threatening spontaneous bleeding. In case of life-threatening bleeding, treatment strategies may include platelets transfusion, IVIG, and glucocorticoids, with the goal of maintaining the platelets at a level to prevent spontaneous bleeding [11].

The onset of ITP and autoimmune thyroid diseases can be highly variable. They may present simultaneously or even during the treatment of one or the 
other disorder [10] [12]. Several reports have been published in this regard highlighting the relation and timings of onset of the two disorders [10] [12]. For instance, Jacobs [13] reported 6 cases of hyperthyroidism and ITP. Out of which 5 developed the two diseases simultaneously, while in 1 patient, thyrotoxicosis occurred after treating thrombocytopenia. On the other hand, a cohort study on thyroid disease in patients with ITP [14] included six patients with ITP and hyperthyroidism, out of which the onset was simultaneous in 1 case, hyperthyroidism occurred first in 3 cases and ITP developed first in 2 cases.

Our patient was also found to be Plasmodium Vivax positive on the 3rd day of admission, and this can lead to thrombocytopenia as well. The cause of Thrombocytopenia in malaria is considered as multifactorial which could be due to antibody-mediated platelet destruction, phagocytosis, bone marrow alterations, coagulation disturbances and splenomegaly [15] [16]. However, only a few studies have documented minor bleeding due to thrombocytopenia in malaria, a majority of the low platelets are not associated with severe bleeding [15] [16] [17]. This case reported bleeding from various sites along with bruises, with a platelet count of $26 \times 10^{\wedge} / 1$ on arrival. Thrombocytopenia can be due to the pathogenesis of both ITP associated with Graves' disease as well as due to malaria. To our knowledge, there is no literature which correlates thrombocytopenia in a patient suffering simultaneously from ITP associated with Grave's disease as well as malaria. However, the added exacerbating effects of malaria on thrombocytopenia may be evident in our case as there was an initial improvement in platelet count after starting antithyroid drug and steroids but dramatic decrease occurred again immediately after malarial infection.

For the treatment of Plasmodium Vivax, chloroquine (CQ) is usually the first -line treatment, followed by 2 weeks of primaquine $(\mathrm{PQ})$, provided the patient is not G6PD deficient [18]. Usually, it is observed that thrombocytopenia following antimalarial treatment gets resolved with a good prognosis [15] [17]. The same management was followed in this case and later on, her malarial parasite came out to be negative, but there was no improvement in symptoms or the platelets count of the patient. This would indicate the severe thrombocytopenia might be mainly due to ITP associated with Grave's disease.

The effect of thyroid disease, its treatment and the course of ITP remains very controversial. There are numerous cases that report resolution of thrombocytopenia, following treatment of hyperthyroidism. However, many cases also report no improvement in thrombocytopenia following an establishment of a euthyroid state [12]. Herman and colleagues [19] reported a case of young women, diagnosed with hyperthyroidism with ITP, whose thrombocytopenia resolved with the treatment of hyperthyroidism. Their survey of the literature till 1978 reported 48 cases of hyperthyroidism and thrombocytopenia. Out of the $22 \mathrm{pa}-$ tients treated for hyperthyroidism, 18 had platelet count returned to normal (80\%) [12] [19]. Gill H and colleagues [1] reported a case of young female diagnosed as ITP associated with Grave's disease, initially the patient was started intravenous immunoglobulin (IVIG) but the patient showed no improvement. 
Then she was started on oral prednisolone but platelets remained low. Carbimazole was then started, with the continuation of prednisolone and patient showed a remarkable increase in platelets count, along with suppression of free T4. Our patient was started on methylprednisolone and carbimazole immediately, and within 3 weeks she also reported normalization of platelet count. Another case [20] of a Chinese woman with autoimmune hyperthyroidism and incidental profound thrombocytopenia was studied and it revealed a drastic improvement in platelet count secondary to the recovery of thyroid symptoms and T4 levels with the concomitant use of carbimazole and prednisolone. However, extended literature search also revealed no durable long-term improvement of thrombocytopenia in patients of ITP associated with autoimmune thyroid conditions, which should be kept in mind while managing such patients [14].

Another aspect of management to be familiar with is the known side effect of thrombocytopenia while administering carbimazole as reported by Jung RS and colleagues [21]. The patient was started on carbimazole for thyrotoxic symptoms, which resolved subsequently, but the platelets count continued to drop for months. Such counter productiveness of carbimazole is not yet seen in our patient, but this is something kept under consideration in the out-patient follow-ups.

\section{Conclusion}

Our case report highlights several important factors. It firstly favors the association between autoimmune thyroid disease and ITP emphasizing the importance of evaluating for thyroid disease in patients with profoundly isolated thrombocytopenia especially those refractory to standard treatment, as evident from literature as well. It also supports the effectiveness of treatment of hyperthyroidism on the normalization of the platelet count in a patient of Graves' disease associated with ITP. However, the long-term improvement monitoring and the adverse effects of anti-thyroid drugs should also be kept in mind while managing and following such patients. Furthermore, our case was also unique in the sense that it documented Plasmodium Vivax malaria as well along with the Graves' disease, both of which have pathogenesis of thrombocytopenia. The literature search in this regard is very sparse.

\section{Consent}

A written informed consent was obtained from the patient's father for this publication.

\section{References}

[1] Gill, H., Hwang, Y.-Y. and Tse, E. (2011) Primary Immune Thrombocytopenia Responding to Antithyroid Treatment in a Patient with Graves' Disease. Annals of Hematology, 90, 223-224. https://doi.org/10.1007/s00277-010-0983-4

[2] Adrouny, A., Sandler, R.M. and Carmel, R. (1982) Variable Presentation of Thrombocytopenia in Graves' Disease. Archives of Internal Medicine, 142, 
1460-1464. https://doi.org/10.1001/archinte.1982.00340210052013

[3] Marshall, J.S., Weisberger, A.S., Levy, R.P. and Breckenridge, R.T. (1967) Coexistent Idiopathic Thrombocytopenic Purpura and Hyperthyroidism. Annals of Internal Medicine, 67, 411-414. https://doi.org/10.7326/0003-4819-67-2-411

[4] Cordiano, I., Betterle, C., Spadaccino, C.A., Soini, B., Girolami, A. and Fabris, F. (1998) Autoimmune Thrombocytopenia (AITP) and Thyroid Autoimmune Disease (TAD): Overlapping Syndromes? Clinical and Experimental Immunology, 113, 373-378. https://doi.org/10.1046/j.1365-2249.1998.00677.x

[5] Brent, G.A. (2008) Graves' Disease. New England Journal of Medicine, 358, 2594-2605. https://doi.org/10.1056/NEJMcp0801880

[6] De Groot, L.J., Dungan, C.G.K., et al. (Eds.) (2000) Diagnosis and Treatment of Graves' Disease. Endotext [Internet] South Dartmouth (MA): MDTextcom, Inc. https://www.ncbi.nlm.nih.gov/books/

[7] Intenzo, C.M., dePapp, A.E., Jabbour, S., Miller, J.L., Kim, S.M. and Capuzzi, D.M. (2003) Scintigraphic Manifestations of Thyrotoxicosis. Radio Graphics, 23, 857-869. https://doi.org/10.1148/rg.234025716

[8] Hou, H., Hu, S., Fan, R., Sun, W., Zhang, X. and Tian, M. (2015) Prognostic Value of $(99 \mathrm{~m}) \mathrm{Tc}$-Pertechnetate Thyroid Scintigraphy in Radioiodine Therapy in a Cohort of Chinese Graves' Disease Patients: A Pilot Clinical Study. BioMed Research International, 2015, Article ID: 974689. https://doi.org/10.1155/2015/974689

[9] Shah, Y. (2011) Thyroid Ophthalmopathy. The Journal of the Association of Physicians of India, 59, 60-65.

[10] Marta, G.N. and de Campos, F.P.F. (2015) Immune Thrombocytopenia and Autoimmune Thyroid Disease: A Controversial Overlap. Autopsy \& Case Reports, 5, 45-48. https://doi.org/10.4322/acr.2015.002

[11] Tahir, H., Sheraz, F., Sagi, J. and Daruwalla, V. (2016) Immune Thrombocytopenia (ITP) Secondary to Subclinical Hashimoto's Thyroiditis: Role of Levothyroxine in Improving the Clinical Outcome of ITP. Journal of Investigative Medicine High Impact Case Reports, 4, 1-4. https://doi.org/10.1177/2324709616647085

[12] Cheung, E. and Liebman, H.A. (2009) Thyroid Disease in Patients with Immune Thrombocytopenia. Hematology/ Oncology Clinics, 23, 1251-1260.

[13] Jacobs, P., Majoos, F. and Perrotta, A. (1984) Hyperthyroidism and Immune Thrombocytopenia. Postgraduate Medical Journal, 60, 657-661. https://doi.org/10.1136/pgmj.60.708.657

[14] Ioachimescu, A.G., Makdissi, A., Lichtin, A. and Zimmerman, R.S. (2007) Thyroid Disease in Patients with Idiopathic Thrombocytopenia: A Cohort Study. Thyroid: Official Journal of the American Thyroid Association, 17, 1137-1142. https://doi.org/10.1089/thy.2007.0066

[15] Lacerda, M.V., Mourao, M.P., Coelho, H.C. and Santos, J.B. (2011) Thrombocytopenia in Malaria: Who Cares? Memorias do Instituto Oswaldo Cruz, 106, 52-63. https://doi.org/10.1590/S0074-02762011000900007

[16] Coelho, H.C.C., Lopes, S.C.P., Pimentel, J.P.D., Nogueira, P.A., Costa, F.T.M., Siqueira, A.M., et al. (2013) Thrombocytopenia in Plasmodium vivax Malaria Is Related to Platelets Phagocytosis. PLoS ONE, 8, e63410. https://doi.org/10.1371/journal.pone.0063410

[17] Kumar, A. and Shashirekha (2006) Thrombocytopenia-An Indicator of Acute Vivax Malaria. Indian Journal of Pathology \& Microbiology, 49, 505-508.

[18] Baird, J.K., Valecha, N., Duparc, S., White, N.J. and Price, R.N. (2016) Diagnosis 
and Treatment of Plasmodium vivax Malaria. The American Journal of Tropical Medicine and Hygiene, 95, 35-51. https://doi.org/10.4269/ajtmh.16-0171

[19] Herman, J., Resnitzky, P. and Fink, A. (1978) Association between Thyrotoxicosis and Thrombocytopenia. A Case Report and Review of the Literature. Israel Journal of Medical Sciences, 14, 469-475.

[20] Yang, W., Ho, B. and Seow, C.J. (2014) Graves' Disease Associated with Immune Thrombocytopenic Purpura: An Uncommon Association with Grave Therapeutic Implications. Endocrine Society, Washington DC.

[21] Jung, R.S., Parghane, R.V., Sood, A., Bhattacharya, A. and Mittal, B.R. (2014) Primary Autoimmune Thrombocytopenia and Co-Existing Graves' Disease: Role of Radioiodine-131. Indian Journal of Nuclear Medicine: The Official Journal of the Society of Nuclear Medicine, India, 29, 195-196.

https://doi.org/10.4103/0972-3919.136602 